\title{
PRE-FRONTAL CORTEX ACTIVITY OF MALE DRIVERS IN THE PRESENCE OF PASSENGERS DURING SIMULATED DRIVING: AN EXPLORATORY FUNCTIONAL NEAR-INFRARED SPECTROSCOPY (fNIRS) STUDY
}

\author{
Anuj K. Pradhan ${ }^{1}$, Xiao-Su (Frank) $\mathrm{Hu}^{2}$, Lisa Buckley ${ }^{1}$, C Raymond Bingham ${ }^{1}$ \\ ${ }^{1}$ University of Michigan Transportation Research Institute \\ ${ }^{2}$ Center for Human Growth and Development \\ University of Michigan, Ann Arbor, MI
}

\begin{abstract}
Summary: Adolescents are more likely to be involved in motor vehicle crashes in the presence of peer passengers, and risky driving behaviors of male teenagers increase in the presence of male peer passengers. There could be several mechanisms of the influence of peer passengers, however it is evident that the male teenage driver with a male peer passenger makes riskier decisions than when alone. The developing teenage brain's activity is different from that of adults during decision-making, especially in regions associated with impulse control, response inhibition, and risk taking. This study tested the feasibility of using functional nearinfrared spectroscopy (fNIRS), a non-invasive brain imaging method that allows in vivo measurements of oxygenated and deoxygenated hemoglobin in cortical tissue, to study drivers' brain activation during simulated driving. Cortical activity was measured in participants driving alone and in the presence of a passenger. When at a dilemma zone at a signalized intersection participants showed increased activation in regions of the left and right medial pre-frontal cortex when driving with a passenger as compared to when driving alone.
\end{abstract}

\section{OBJECTIVES}

Motor vehicle crashes are the leading cause of mortality, and a major cause of injury for U.S. teenagers (CDC, 2012). This risk is higher for male than female teenage drivers, and even greater when male teenage passengers are present (Ouimet et al., 2010). Why and how teenage passengers result in increased crash risk is not well understood. The study of brain activity is a promising means of examining this passenger effect on crash risk. Findings from neural imaging studies suggest that the presence of peers increases adolescent risk taking by heightening reward sensitivity (Chein et al, 2011). Neuroimaging studies examining peer influence on teenagers have primarily been conducted using functional magnetic resonance imaging (fMRI) (Chein et al, 2011; Schweizer et al, 2013). However, fMRI limits the study of driving behavior in more realistic conditions, such as in driving simulators. fMRI requires that the subject be lying down, limit movement, and must be conducted in the absence of metallic objects, thereby imposing limitations on the ecological validity of experimental conditions. In contrast, functional nearinfrared spectroscopy (fNIRS) is a less restrictive and a non-invasive imaging technique that uses near-infrared light to measure brain activation. While fNIRS has lower spatial resolution for imaging than fMRI, it has a higher temporal resolution allowing continuous monitoring of changes in brain activity. fNIRS uses headgear outfitted with sensors and detectors that can be worn comfortably by a subject while performing tasks normally, making this a brain imaging technique in more ecologically-valid conditions (Kojima et al, 2005). This exploratory study 
tested the feasibility of fNIRS as a technique to monitor drivers' brain activity during simulated driving, thus testing an innovative approach to driver behavior measurement.

\section{METHODS}

\section{Participants}

Twelve young male adults (25-29 years) were recruited. They all had a regular driver license, drove at least twice a week, and had been licensed for at least 12 months. They were screened according to criteria such as being neurotypical (no diagnosed neurological conditions), righthanded, not wearing prescription glasses, not currently taking any psychoactive medication, and not prone to simulator sickness. Participants were provided a $\$ 50$ incentive for the 1.5 hour visit.

\section{Equipment}

Driving Simulator. An RTI (Realtime Technologies Inc.) desktop driving simulator was used for the study. The simulator consists of a widescreen monitor, a steering wheel, and foot pedals for braking and acceleration, runs RTI's simulation engine, SimCreator, and is highly programmable to create virtual driving worlds and scenarios and to collect driving data at $30 \mathrm{~Hz}$. The desktop system was chosen due to restrictions on the transport of fNIRS equipment to a full-cab driving simulator laboratory, but helps in establishing proof of concept and feasibility.

Functional Near-Infrared Spectroscopy (fNIRS): NIRS is an optical method of non-invasively measuring brain activity as indicated by oxygenation of brain tissue (Bunce et al, 2006). Brain activity is fueled by glucose metabolism so increased neural activity increases glucose and oxygen consumption. This results in increased local cerebral blood flow that carries glucose and oxygen to the active brain areas. The oxygen is transported by oxygenated hemoglobin $(\mathrm{O} 2 \mathrm{Hb})$ in the blood, and as oxygen is withdrawn for metabolism of glucose there is an increase in the volume of deoxygenated hemoglobin $(\mathrm{HHb})$. The optical properties of $\mathrm{O} 2 \mathrm{Hb}$ and $\mathrm{HHb}$ in the near-infrared light range $(700-900 \mathrm{~nm})$ make it possible to measure change in their concentration using optical methods, i.e. fNIRS (Obrig et al, 2000). These methods have been successfully employed to study brain activation associated with attention (Derosiere et al, 2013) and working memory (Ehlis et al, 2008); with cognitive tasks such as verbal-fluency task (Herrman et al, 2003) and the Stroop task (Serap et al, 2009); and decision making behavior tasks such as the Balloon Analog Risk Task (BART) (Li et al, 2013).

This study used a TechEn CW6 fNIRS system with 690 and $830 \mathrm{~nm}$ wavelengths to measure hemodynamic changes in the drivers' brains. This system uses a continuous wave approach incorporating up to 32 lasers and detectors, and provides real-time data acquisition and display of raw signals for each wavelength and laser/detector combination. The system also provides connections for auxiliary signals and triggers for external devices facilitating integration with the driving simulator. The equipment provides low profile, low weight optical probes and fibers that allow quick and comfortable setup for participants. Headgear was customized for this study based on imaging of specific brain regions. The regions of interest (ROI) of the brain were determined based on previous studies of functional activation. Since fNIRS measurements is limited to the cortical surface and the limited size of the probes and headgear have additional 
constraints, in this study the ROI were chosen to encompass the prefrontal cortex (PFC) including the ventrolateral prefrontal cortex (VLPFC), the dorsolateral prefrontal cortex (DLPFC), and the orbitofrontal cortex (OFC), based on their roles in response inhibition (Herrmann et al, 2005), incentive processing and cognitive control (Chein et al, 2011), and risky decision making (Cazzell et al, 2012; Chen et al, 2015). The final headgear included 7 emitters and 7 detectors spaced $3 \mathrm{~cm}$ apart, yielding 19 data channels sampled at $50 \mathrm{~Hz}$. Optodes were mounted into a custom-3D-printed headband. Figure 1 shows the probe configuration (letters indicate emitters, numbers indicate detectors) and the placement of the headband on the subject. Brain activation was examined in bilateral prefrontal cortex regions. The probe localization was established and applied consistently for each participant using the international 10-10 transcranial system positioning (AES, 1994). Fz, Cz locations were measured for each participant as anchor points.

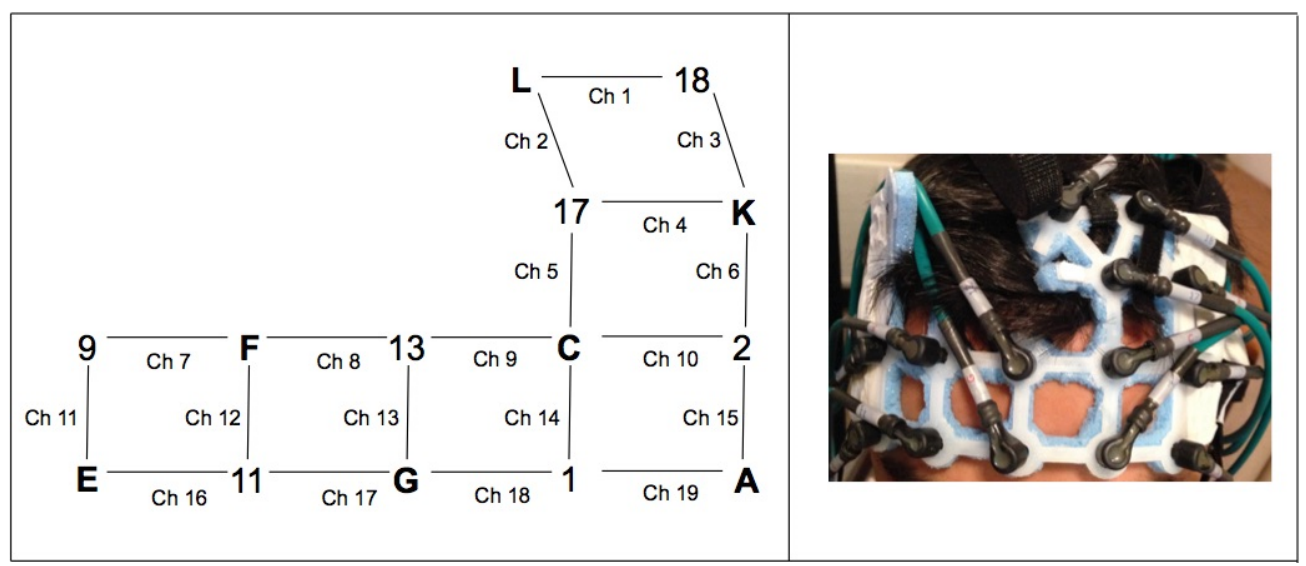

Figure 1. Probe configuration (with channels numbered) and 3-D printed headband

\section{Procedure}

The study employed a within-subject design with each participant encountering two driving conditions, solo- \& passenger-drive. To control for order-effects the drive orders (solo-first vs. passenger-first) were counterbalanced, and to minimize carryover effects (of passenger presence) a washout task (word search task) was given to participants between drives. A male confederate portrayed the role of a peer-aged passenger in order to maintain experimental control. The University of Michigan IRB approved the experimental protocol, which was based on previous successful passenger condition driving simulation experiments (Simons-Morton et al, 2013).

Each participant started his visit by signing a consent form. He was then outfitted with the fNIRS sensors, a process that included head measurements, fitting of the headgear, and the placement of optodes on appropriate regions on the prefrontal cortex according to a modified international EEG 10-10 system (AES, 1994). After sensor calibration (including ensuring signal detection), the participant was familiarized with the driving simulator via a practice drive. The simulator was programmed to provide an environment that would elicit natural driving behavior. Interspersed within the drives were ten scenarios (events) that required decisions regarding risks, specifically, dilemma-zone yellow lights presented at four-way signalized intersections. The total 
driving time did not exceed 30 minutes, reducing potential discomfort due to simulator sickness or optodes. Participants' brain activity was recorded simultaneously during the drives.

There is evidence that the mere presence of a peer may influence the driving behavior of teenagers (Simons-Morton et al, 2013). For the purposes of this exploratory study the confederate was trained to solely maintain a presence as a passenger without engaging the driver. The confederate ruse was also based on previously successful procedures (Simons-Morton et al, 2013). The participant was told that the confederate had also been recruited as a participant, but since he had to wait for "another" simulator to be available the experimenter would like the confederate to sit with the participant during one of the two experimental drives in order to observe simulator operation. Both participant and confederate were instructed to minimize interactions during the drives. The confederate sat to the right of the participant and could view the entire drive. The participant's task was to safely drive to a destination as guided by street signs. The participants were told that the base incentive payment would start at $\$ 40$ and reaching the destination earlier could increase payments up to a maximum total of $\$ 50$. However breaking road rules (e.g., running red lights, speeding) could result in deductions to no less than $\$ 30$. Participants were also told that they could make up for lost points by good driving. However, for ethical purposes all participants were paid \$50. On inquiries, the researchers informed participants that the driving score was automatically computed.

\section{ANALYSES AND RESULTS}

The outcome measure of interest from the simulator was driver behavior at the yellow dilemma zone intersections (events), i.e., whether a driver stopped or not at a yellow light. There were no significant differences in the stopping behaviors between the solo and passenger-drive conditions (percentage stopped $=0.11$ with passenger; $=0.15$ without passenger; $p=0.10$ ).

The outcome measure from fNIRS was neural activity measured by changes in concentration of $\mathrm{O} 2 \mathrm{Hb} \& \mathrm{HHb}$ during specific events in the simulation at specified prefrontal cortex areas. Neural activity was analyzed for each event before the driver saw a yellow signal. Imaging data were preprocessed using Homer2 (Huppert et al, 2009), a MATLAB-based software. The preprocessing steps were performed in the following order: data pre-examination, optical density change data conversion, data de-trending, filtering, and concentration change data conversion. Participants with missing simulator data or those who did not complete the experiment were excluded from analyses. (Of the 12 participants, data from 9 were retained for analysis). The raw time course data were then converted into units of optical density change $(\Delta \mathrm{OD})$. Data then went through a detrending process. Finally, a low-pass filter with cutoff frequency at $0.5 \mathrm{~Hz}$ was applied to the $\triangle \mathrm{OD}$ data and the hemoglobin concentration change data was calculated using the modified Beer-Lambert law, which yielded $\mathrm{O} 2 \mathrm{Hb}$ and $\mathrm{HHb}$ values. Only $\mathrm{O} 2 \mathrm{Hb}$ values were analyzed in this study, due to previous evidence showing its consistency in revealing brain activation (Lin et al, 2009; Xiao-Su et al, 2010).

Each participant's hemoglobin concentration data from multiple events from two driving sessions (solo- and passenger-) were analyzed using an event-averaging algorithm based on stimulus markers programmed at each event. The time range selected for averaging started 6seconds before an intersection was reached (this window incorporated the onset of the yellow 
light) and was 16 seconds long to allow for sufficient measurement of changes in blood flow. Finally, the derived averaged time series from two driving sessions were compared using a twotailed t-test $(p<0.05)$. The analyses showed statistically significant differences in brain activation levels in various ROI based on passenger condition. In particular, when the light turned yellow, drivers showed higher brain activity in the left medial prefrontal cortex (Channels $7,8,12$ ) and right medial prefrontal cortex (Channels 9,10,14,15) while driving with a passenger as compared to driving alone (See Figure 2 for example).

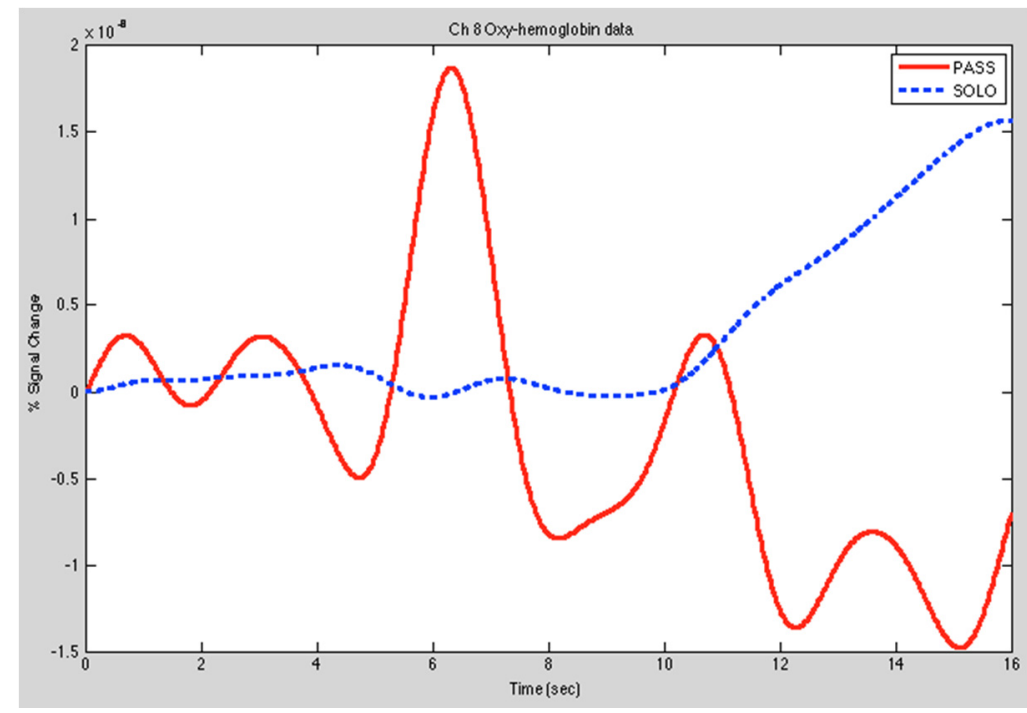

Figure 2. Averaged hemodynamic response for Channel 8

\section{CONCLUSION}

This study used fNIRS technology to measure brain activity in the pre-frontal cortex of drivers during a simulated driving task, with and without a passenger. The results show that drivers did not behave differently, i.e., they did not stop less or more frequently, at yellow intersections regardless of whether they drove alone or with a passenger. However, the neural data show significantly higher activation in the left and right medial prefrontal cortices when approaching a yellow light when the participants drove with a passenger. Studies have shown these areas to be associated with active risky decision-making (Rao et al, 2008), voluntary decision-making (Cazzell et al, 2012), and response inhibition (Rubia et al, 2003). Thus, despite no outward (simulator-measured) behavioral evidence of specific driving decisions, the data suggest active differences in neural processing during these scenarios. No data are available from prior experimental fNIRS research in the driving domain on which to base directionality of effects or how that might vary across brain regions in terms of risk taking or age characteristics. However, neuroscience research on teenage brain development has shown functional differences when compared to adults in the regions that were examined in this study. Further fNIRS experiments can extend those findings. Despite limitations of small sample size and low ecological validity, the result is promising and offers new insight into neural activation in adults based on passenger condition. This study tested the feasibility of using fNIRS to measure brain activation in a simulated driving environment. The findings establish feasibility of this approach to studying driver behavior, including selection of brain ROI and understanding potential limitations; thus, it 
lays the groundwork for further research with higher levels of ecological validity, including testtrack or naturalistic driving. These findings provide foundational evidence that can be used in future studies of neural activation in drivers, especially adolescents. The results of this study support the use of fNIRS in studying driver behavior, and provide critical pilot data that support larger scale studies of driver risk using this methodology and approach.

\section{ACKNOWLEDGMENTS}

The authors gratefully acknowledge Dr. Silvia Bisconti, Hannah Clark, Margaret Ugolini, and other members of the Univ of Michigan Transportation Research Institute (UMTRI) and the fNIRS Laboratory at the Center for Human Growth \& Development (CHGD) at the Univ of Michigan. This research is supported by the Rauner Family Foundation Grants from CHGD, and from the Center for Advancing Transportation Leadership \& Safety (ATLAS), a collaborative UTC between the UMTRI and the Texas A\&M Transportation Institute. The ATLAS Center is sponsored by USDOT's Research \& Innovative Technology Administration (RITA) through Grant Number DTRT13-G-UTC54. The contents of this report reflect the views of the authors, who are responsible for the facts and the accuracy of the information presented. This document is disseminated under the sponsorship of the USDOT's UTC Program in the interest of information exchange. The U.S. government assumes no liability for the contents or use thereof.

\section{REFERENCES}

American Electroencephalographic Society. 1994. Guideline thirteen: guidelines for standard electrode position nomenclature. J Clin Neurophysiol. 11:111-113.

Bunce, S. C., Izzetoglu, M., Izzetoglu, K., Onaral, B., \& Pourrezaei, K. (2006). Functional nearinfrared spectroscopy. IEEE engineering in medicine and biology magazine: the quarterly magazine of the Engineering in Medicine \& Biology Society, 25(4), 54-62.

Cazzell, M., Li, L., Lin, J.Z., Patel, S.J. \& Liu, H. (2012), “Comparison of neural correlates of risk decision making between genders: an exploratory fNIRS study of the Balloon Analogue Risk Task (BART)," Neuroimage, 62(3), 1896-911

Centers for Disease Control and Prevention, \& National Center for Injury Prevention and Control. (2012). Web-based Injury Statistics Query and Reporting System (WISQARS). http://www.cdc.gov/injury/wisqars/index.html.

Chein, J. M., Albert, D., O'Brien, L., Uckert, K., \& Steinberg, L. (2011). Peers increase adolescent risk taking by enhancing activity in the brain's reward circuitry. Developmental Science, 14(2), F1-F10.

Chen, K.-H., Rusch, M. L., Dawson, J. D., Rizzo, M., \& Anderson, S. W. (2015). Susceptibility to social pressure following ventromedial Social Cognitive and Affective Neuroscience.

Derosière, G., Billot, M., Ward, E. T., \& Perrey, S. (2013). Adaptations of Motor Neural Structures' Activity to Lapses in Attention. Cerebral cortex (New York, N.Y.!: 1991), 1-9.

Ehlis, A.-C., Bähne, C. G., Jacob, C. P., Herrmann, M. J., \& Fallgatter, A. J. (2008). Reduced lateral prefrontal activation in adult patients with attention-deficit/hyperactivity disorder 
(ADHD) during a working memory task: a functional near-infrared spectroscopy (fNIRS) study. Journal of psychiatric research, 42(13), 1060-7.

Herrmann, M. J., Ehlis, A.C., \& Fallgatter, A. J. (2003). Frontal activation during a verbalfluency task as measured by near-infrared spectroscopy.Brain Research Bulletin,61(1),51-56.

Herrmann MJ, Plichta MM, Ehlis AC, Fallgatter AJ.(2005) Optical topography during a GoNoGo task assessed with multi-channel near-infrared spectroscopy. Behav Brain Res $2005 ; 160: 135-40$

Huppert, T.J., Diamond, S.G., Franceschini, M.A.,Boas, D.A. (2009). HomER:a review of timeseries analysis methods for near-infrared spectroscopy of the brain. Appl Opt 48,10:D280-98.

Kojima, T., Tsunashima, H., Shiozawa, T., \& Takada, H. (2005). Measurement of train driver's brain activity by functional near-infrared spectroscopy (fNIRS).The Second Asian and Pacific Rim Symposium on Biophotonics, 2004. APBP 2004.

Li, L., Lin, Z., Cazzell, M., \& Liu, H. (2013). Test-retest assessment of functional near-infrared spectroscopy to measure risk decision making in young adults. In N. Kollias, B. Choi, H. Zeng, H. W. Kang, B. E. Knudsen, B. J. Wong, ... M. D. Morris (Eds.), Proc. of SPIE (Vol. 8565, pp. 856557-1-7).

Lin, PY, Lin, SI, Penney, T, Chen J. (2009) Review: applications of near infrared spectroscopy and imaging for motor rehabilitation in stroke patients. J Med Biol Eng, 2009

Obrig, H., Wenzel, R., Kohl, M., Horst, S., Wobst, P., Steinbring, J., Thomas, F., Villringer, A., (2000) Near-infrared spectroscopy: does it function in functional activation studies of the adult brain, Int. J. Psychophysiol. 35, 125-142.

Ouimet, M. C., Simons-Morton, B. G., Zador, P. L., Lerner, N. D., Freedman, M., Duncan, G. D., \& Wang, J. (2010). Using the U.S. National Household Travel Survey to estimate the impact of passenger characteristics on young drivers' relative risk of fatal crash involvement. Accident Analysis \& Prevention, 42, 689-694.

Rao, H., Korczykowski, M., Pluta, J., Hoang, A., \& Detre, J. A. (2008). Neural correlates of voluntary and involuntary risk taking in the human brain: an fMRI Study of the Balloon Analog Risk Task (BART). Neuroimage, 42(2), 902-910.

Rubia, K., Smith, A. B., Brammer, M. J., \& Taylor, E. (2003). Right inferior prefrontal cortex mediates response inhibition while mesial prefrontal cortex is responsible for error detection. Neuroimage, 20(1), 351-358.

Schweizer, T., Kan, K., Hung, Y., Tam, F., Naglie, G. \& Graham, S. J. (2013) Brain activity during driving with distraction: an immersive fMRI study. Fron in hum neuroscience, 7, 53

Serap, S., Tapsin, M., \& Akin, A. (2009). Investigating brain hemodynamics of ADHD patients by functional near infrared spectroscopy. Annual International Conference of the IEEE Engineering in Medicine and Biology Society. IEEE Engineering in Medicine and Biology Society. Conference, 2009, 3028-30.

Simons-Morton, B.G., Bingham, C.R., Falk, E.B., Li, K., Pradhan, A.K., Ouimet, M.C., Almani, F., \& Shope, J.T. (2013) Experimental Effects of Injunctive Norms on Simulated Risky Driving Among Teenage Males. Health Psychology

Xiao-Su, H., Keum-Shik H., Shuzhi S. G., Myung-Yung, J., (2010) Kalman estimator- and general linear model-based on-line brain activation mapping by near-infrared spectroscopy. BioMedical Engineering OnLine 2010 9:82. 